\title{
A review article on ovarian cystectomy in endometriomas by laparoscopy
}

\author{
Amita Yadav*, Shweta Patel
}

Department of Obstetrics and Gynecology, MLN Medical College, Allahabad, Uttar Pradesh, India

Received: 10 September 2017

Accepted: 05 October 2017

\section{*Correspondence:}

Dr. Amita Yadav,

E-mail: amitayadav0212@gmail.com

Copyright: (c) the author(s), publisher and licensee Medip Academy. This is an open-access article distributed under the terms of the Creative Commons Attribution Non-Commercial License, which permits unrestricted non-commercial use, distribution, and reproduction in any medium, provided the original work is properly cited.

\begin{abstract}
Endometrioma is one of the most frequent adnexal masses in the reproductive population. Medical therapy is inefficient and cannot be recommended in the management of ovarian endometriomas. The general consensus is that ovarian endometriomas larger than $4 \mathrm{~cm}$ should be removed, both to reduce pain and to improve spontaneous conception rates. The removal of ovarian endometriomas can be difficult, as the capsule is often densely adherent. While the surgical treatment of choice is surgical laparoscopy, for conservative treatment, the preferred method is modified combined cystectomy. Cystectomy can be destructive for the ovary, whereas ablation may be incomplete, with a greater risk of recurrence.
\end{abstract}

Keywords: Combined technique, Cystectomy, Endometrioma, Laparoscopy

\section{INTRODUCTION}

The ovaries are a common site for endometriosis. Endometrioma is one of the most frequent adnexal masses. Although endometrioma is the most frequent ovarian mass that is encountered by gynaecologists, there are still controversies on its pathogenesis, risk of malignant transformation, modalities of treatment, and effect on fertility.

Laparoscopy is extremely useful in the diagnosis and treatment of endometriomas.

\section{DIAGNOSIS}

The definitive diagnosis of the endometriosis is made by visualizing lesions during surgery and obtaining histological confirmation. Transvaginal sonography has been found to be a good test for assessing the severity of pelvic endometriosis. The morphology of ovarian endometriomas in the ultrasonography examination reveals a round, homogenous, hypoechoic cyst with or without internal septa and with poor vascularization of the cyst wall. Diffuse low-level internal echoes occur in $95 \%$ of endometriomas. Endometriomas are usually adherent to the pelvic side wall. This immobility is a useful diagnostic indicator.

Lower anti-Mullerian hormone serum levels and an association with the severity were found in women with endometriosis. This information might be useful in patients, especially those with severe endometriosis undergoing controlled ovarian stimulation. ${ }^{1}$ Putative serum markers (monocyte chemoattractant protein 1, migration inhibitory factor, leptin, and CA-125) improved their diagnostic capability to $73 \%$ of patients, with $94 \%$ overall accuracy. ${ }^{2}$

A new marker, HE4 (human epididymis secretory protein-4), could be used in the differential diagnosis of endometriosis cyst. Human epidydimal secretory protein $\mathrm{E} 4$ is a new promising biomarker for ovarian cancer. The combination of HE4 and CA 125 assay could discriminate ovarian endometriosis cysts from malignant 
ovarian tumors effectively. ${ }^{3}$ The advantage of HE4 over CA125 is mainly in the detection of borderline ovarian tumors and early-stage epithelial ovarian and tubal cancers. ${ }^{4}$ A normal HE4 value in this situation would imply the differential diagnosis of a benign endometrioma rather than ovarian cancer, and this patient could therefore be operated on laparoscopically by a gynecologist. Both markers showed similar diagnostic performance in the detection of epithelial ovarian cancer at clinically defined thresholds (CA125 $35 \mathrm{U} / \mathrm{mL}$; HE4 $70 \mathrm{pM}$ ), but HE4 was not elevated in endometriosis. ${ }^{5}$ An analysis of serum HE4 concentrations together with a tumor marker, CA125, in serum samples of women diagnosed with various types of endometriosis, endometrial cancer, or ovarian cancer and in samples from healthy controls has been released. ${ }^{6}$ Based on this study, the mean serum concentration of HE4 was significantly higher in serum samples of patients with both endometrial (99.2 pM, p<0.001) and ovarian (1125.4 $\mathrm{pM}, \mathrm{p}<0.001)$ cancer but not with ovarian endometriomas (46.0 pM) or other types of endometriosis $(45.5 \mathrm{pM})$ as compared with healthy controls (40.5 pM). Serum HE4 concentration is a valuable marker to better distinguish patients with ovarian malignancies from those suffering from benign ovarian endometriotic cysts.

Laparoscopy has the advantage of allowing simultaneous diagnosis, staging, and treatment of ovarian endometriosis.

\section{LAPAROSCOPY FOR ENDOMETRIOMAS}

\section{Indications for surgery}

Five specific complications may be associated with the non-surgical approach:

- Risk of causing rupture of the endometrioma and/or the development of a pelvic abscess

- Missing an occult early-stage malignancy

- Difficulties during oocyte retrieval

- Follicular fluid contamination with endometrioma content

- $\quad$ Progression of endometriosis. ${ }^{7}$

Endometriosis is shown to increase the risk of certain subtypes of ovarian cancer, such as endometrioid and clear-cell carcinomas. ${ }^{8}$ There are data indicating that $40 \%$ of endometrioid ovarian carcinomas and $50 \%$ of clearcell ovarian carcinomas are associated with endometriosis. Both endometrioid and clear-cell carcinomas are thought to arise, at least partly, from endometriosis. Similar pathophysiological mechanisms may be involved in the progression of endometriosis, as well as in its transformation into ovarian neoplasia. ${ }^{9}$

However, medical treatment for the endometrioma does not improve fertility. Surgical treatment is effective in the treatment of pain and fertility, particularly for women with more severe endometriosis. It improves pelvic pain and deep dyspareunia. Draining the endometrioma or partially resecting its wall is inadequate, because the endometrial tissue lining the cyst can remain functional and may cause the symptoms to recur. Cystectomy of ovarian endometriomas improves spontaneous pregnancy rates and reduces pain. It has been shown that laparoscopic cystectomy for ovarian endometriomas $>4$ $\mathrm{cm}$ in diameter improves fertility compared to drainage and coagulation. ${ }^{11}$

Laparoscopy has the advantage of allowing simultaneous diagnosis, staging, and treatment of ovarian endometriosis. The main surgical procedures for treatment of endometrioma include ultrasound-guided or laparoscopy-guided aspiration, laparoscopic surgery by means of cystectomy or fenestration and coagulation, radical-treatment ovariectomy or adnexectomy. ${ }^{12}$

\section{Surgical technique}

A general consensus is that ovarian endometriomas larger than $4 \mathrm{~cm}$ should be removed, both to reduce pain and to improve spontaneous conception rates. The presence of small endometriomas $(2-4 \mathrm{~cm})$ does not reduce the success of in vitro fertilization (IVF) treatment. ${ }^{13}$ However, all decisions to operate a cyst beyond 3 or $4 \mathrm{~cm}$ are arbitrary, as there is no evidence to support one or the other. ${ }^{10}$ Surgeons should bear in mind that if all healthy growing follicles may be reached without damaging the endometrioma, a cyst over 4 or even $5 \mathrm{~cm}$ does not require surgery in asymptomatic patients.

The goal of operative treatment of endometriosis is to remove all implants, resect adhesions, relieve pain, reduce the risk of recurrence and postoperative adhesions, and restore the involved organs to a normal anatomic and physiologic condition. endometriomas must be treated by laparoscopy. Laparoscopic cystectomy remains a firstline choice for the conservative treatment of endometriotic cysts.

In this study, instillation of endometrioma fluid, followed by copious saline lavage, is strongly associated with adhesion formation. ${ }^{14}$

It has been known that surgical resection of endometriomas, either by laparotomy or laparoscopy, is equally effective in eradicating endometriosis; however, if resection of endometriomas is not sufficiently radical, the risk of recurrence is high.

\section{Fenestration and ablation (fulguration or vaporization)}

Endometrial implants or endometriomas less than $2 \mathrm{~cm}$ in diameter are coagulated, laser-ablated, or excised using scissors, biopsy forceps, laser, or electrodes. For successful eradications, all visible lesions and scars must be removed from the ovarian surface. A major problem with laparoscopic cyst drainage is a high risk of recurrence of about $80 \%$ to $100 \%$. $^{15}$ 
An alternative method is to perform laparoscopic cyst fenestration and ablation of the cyst capsule. This has been shown to significantly improve pelvic pain, with a very high patient satisfaction rating. Fenestration and ablation or laser vaporization of endometriomas without excision of the pseudocapsule is associated with a significantly increased risk of cyst recurrence.

Alborzi et al found that laparoscopic ovarian cystectomy was associated with better outcomes in pregnancy rates and relief of pain than fenestration and coagulation of endometriomas and had a lower rate of reoperation, even in larger cysts. ${ }^{16}$

Laparoscopic cystectomy remains the first-line choice for conservative treatment of endometriotic cysts.

Another method involves hydrodissection of the plane between the cyst wall and the ovarian stroma. ${ }^{17}$

Canis et al and Marconi et al found no effect on ovarian response in IVF after cystectomy. ${ }^{18,19}$ But, there are many other trials that indicate that cystectomy has a bad influence in the subsequent ovarian responsiveness. As Hart et al. concluded, excisional surgery of endometriomas results in a more favorable outcome than drainage and ablation in terms of recurrence, pain symptoms, subsequent spontaneous pregnancy in previously sub-fertile women, and ovarian response to stimulation. ${ }^{20}$ But, Muzii et al showed that recognizable ovarian tissue was inadvertently excised together with the endometriotic cyst wall in most cases during stripping for endometrioma excision. ${ }^{21}$ Close to the ovarian hilus, ovarian tissue that was removed along the endometrioma wall contained primordial, primary, and secondary follicles in $69 \%$ of cases. Away from the hilus, no follicles or only primordial follicles were found in $60 \%$ of specimens. There is obviously an absence of a clear plane of cleavage close to the ovarian hilus. However, it has been published by the same group that the ovarian tissue adjacent to the endometrioma wall differs morphologically from normal ovarian tissue; it never shows the follicular pattern that is observed in normal ovaries. $^{22}$

Nargund et al showed that, in cycles with ovulation induction after cystectomy, revealed less follicular count in the ovary that underwent surgical excision. ${ }^{23}$ Ho et al concluded that surgery for ovarian endometriomas induces a poor ovarian response to controlled ovarian hyperstimulation. ${ }^{24}$ Laparoscopic cystectomy has been questioned with respect to damage to the operated ovary.

The risk of excessive surgery (removal or destruction of normal ovarian cortex together with the endometrioma) and the risk of incomplete surgery (with subsequent early recurrence of endometriomas). ${ }^{25}$ To overcome these problems, Donnez et al described a new mixed technique for the laparoscopic management of endometriomas. ${ }^{26}$
Electrosurgical coagulation of the remaining parenchyma after excision of the cyst wall may cause further damage to the ovarian tissue. However, when appropriate techniques are used, small vessels may be identified and coagulated with bipolar forceps that, under these circumstances, may limit thermal damage to less than 0.2 $\mathrm{mm} \cdot{ }^{27}$

Unfortunately, pelvic surgery for endometriosis has been associated with high rates of adhesion formation and reformation. $^{29}$ After laparoscopic endometriosis surgery, the odds of adhesion formation range from $80 \%$ to $100 \% .^{30}$ The high incidence of adhesion formation after surgery for endometriosis underscores the importance of optimizing the surgical technique and the possible role of anti-adhesion drugs to potentially reduce adhesion formation. To reduce postoperative adhesion, we prefer to use adhesion barrier gels (Hyalobarrier Gel; The Nordic Group, Paris France).

\section{CONCLUSION}

Medical therapy is inefficient and cannot be recommended in the management of ovarian endometriomas. While the surgical treatment of choice is surgical laparoscopy, for conservative treatment, the preferred method is modified combined cystectomy. There are no indications for systematic pre-operative medical treatment to facilitate the cystectomy. Postoperative medical treatment has no benefits in cases of infertility. But, treatment of the endometriosis, as well as endometriomas, must be individualized, taking the clinical problem in its entirety into account, including the impact of the disease and the effect of its treatment on quality of life.

The excision of the cyst wall in endometriomas is strongly recommended, especially in infertile patients. However, in light of all of this evidence, we can conclude that cystectomy may be destructive to the ovary. On the contrary, inefficient coagulation may create a great risk of recurrence.

It is essential to minimize or avoid spillage of endometrioma contents during surgical resection and thereby avoid the need for irrigation and suction. Use of careful dissection, aspiration of the endometrioma fluid before rupture, and a sterile bag collection device to avoid spillage of the endometrioma fluid are possible substitute techniques to the currently accepted surgical approach.

It has been shown that the stripping procedure used in laparoscopy for ovarian cyst excision appears to be an organ-preserving procedure. Besides, in nonendometriotic cysts, some ovarian tissue was inadvertently excised with the cyst wall in the endometriotic cysts. These specimens included some ovarian tissue, approximately $1-2 \mathrm{~mm}$ in thickness, excised along with the cyst pseudocapsule; this tissue, 
however, did not show the morphologic characteristics seen in normal ovarian tissue.

Laparoscopic ovarian cystectomy is recommended if an ovarian endometrioma $\geq 4 \mathrm{~cm}$ in diameter is present to confirm the diagnosis histologically, reduce the risk of infection, improve access to follicles, and possibly improve ovarian response.

Funding: No funding sources Conflict of interest: None declared

Ethical approval: Not required

\section{REFERENCES}

1. Shebl O, Ebner T, Sommergruber M, Sir A, Tews G. Anti-Muellerian hormone serum levels in women with endometriosis: a case-control study. Gynecol Endocrinol. 2009;25:713-6.

2. Adamson GD. Endometriosis classification: An update. Curr Opin Obstet Gynecol. 2011;23:213-20.

3. Montagnana M, Lippi G, Danese E, Franchi M, Guidi GC. Usefulness of serum HE4 in endometriotic cysts. Br J Cancer. 2009;101:548-8.

4. Moore RG, Jabre-Raughley M, Brown AK, Robison KM, Miller MC, Allard WJ, et al. Comparison of a novel multiple marker assay versus the risk of malignancy index for the prediction of epithelial ovarian cancer in patients with a pelvic mass. Am J Obstet Gynecol. 2010;203:228-33.

5. Jacob F, Meier M, Caduff R, Goldstein D, Pochechueva T, Hacker N, et al. No benefit from combining HE4 and CA125 as ovarian tumor markers in a clinical setting. Gynecol Oncol. 2011;121:487-91.

6. Huhtinen K, Suvitie P, Hiissa J, Junnila J, Huvila J, Kujari $H$, et al. Serum HE4 concentration differentiates malignant ovarian tumors from ovarian endometriotic cysts. Br J Cancer. 2009;100:1315-9.

7. Somigliana E, Vercellini P, Viganó P, Ragni G, Crosignani PG. Should endometriomas be treated before IVF-ICSI cycles? Hum Reprod Update. 2006;12:57-64.

8. Nagle C, Olsen C, Webb P, Jordan S, Whiteman D, Green A. Endometrioid and clear cell ovarian cancers: a comparative analysis of risk factors. Eur J Cancer. 2008;44:2477-84

9. Ness R. Endometriosis and ovarian cancer: thoughts on shared pathophysiology. Am J Obstet Gynecol. 2003;189:280-94.

10. Garcia-Velasco JA, Somigliana E. Management of endometriomas in women requiring IVF: to touch or not to touch. Hum Reprod. 2009;24:496-501.

11. Kennedy S, Bergqvist A, Chapron C, D'Hooghe T, Dunselman G, Greb R, et al. ESHRE guideline for the diagnosis and treatment of endometriosis. Hum Reprod. 2005;20:2698-704

12. Chapron C, Vercellini P, Barakat H, Vieira M, Dubuisson JB. Management of ovarian endometriomas. Hum Reprod Update. 2002;8:591-7.
13. Tinkanen $\mathrm{H}$, Kujansuu E. In vitro fertilisation in patients with ovarian endometriosis. Acta Obstet Gynecol Scand. 2000;79:119-22.

14. Smith LP, Williams CD, Doyle JO, Closshey WB, Brix WK, Pastore LM. Effect of endometrioma cyst fluid exposure on peritoneal adhesion formation in a rabbit model. Fertil Steril. 2007;87:1173-9.

15. Marana R, Caruana P, Muzii L, Catalano GF, Mancuso S. Operative laparoscopy for ovarian cysts excision versus aspiration. $\mathbf{J}$ Reprod Med. 1996;41:435-8.

16. Alborzi S, Ravanbakhsh R, Parsanezhad ME, Alborzi M, Alborzi S, Dehbashi S. A comparison of follicular response of ovaries to ovulation induction after laparoscopic ovarian cystectomy or fenestration and coagulation versus normal ovaries in patients with endometrioma. Fertil Steril. 2007;88:507-9.

17. Nezhat C, Silfen SL, Nezhat F. Surgery for endometriosis. Curr Opin Obstet Gynecol. 1991;3:385-90.

18. Canis M, Pouly JL, Tamburro S, Mage G, Wattiez A, Bruhat MA. Ovarian response during IVF-embryo transfer cycles after laparoscopic ovarian cystectomy for endometriotic cysts of $>3 \mathrm{~cm}$ in diameter. Hum Reprod. 2001;12:2583-6.

19. Marconi G, Vilela M, Quintana R, Sueldo C. Laparoscopic ovarian cystectomy of endometriomas does not affect the ovarian response to gonadotropin stimulation. Fertil Steril. 2002;78:876-8.

20. Hart R, Hickey M, Maouris P, Buckett W. Excisional surgery versus ablative surgery for ovarian endometriomata. Cochrane Database Syst Rev. 2008;16:CD004992.

21. Muzii L, Bellati F, Bianchi A, Palaia I, Manci N, Zullo $\mathrm{M}$, et al. Laparoscopic stripping of endometriomas: a randomized trial on different surgical techniques. Part II. Pathological results. Hum Reprod. 2005;20:1987-92.

22. Muzii L, Bianchi A, Crocè C, Manci N, Panici PB. Laparoscopic excision of ovarian cysts: is the stripping technique a tissue-sparing procedure? Fertil Steril. 2002;77:609-14.

23. Nargund G, Cheng W, Parsons J. The impact of ovarian cystectomy on ovarian response to stimulation during in-vitro fertilization cycles. Hum Reprod. 1996;11:81-3.

24. Ho H, Lee R, Hwu Y, Lin M, Su J, Tsai Y. Poor response of ovaries with endometrioma previously treated with cystectomy to controlled ovarian hyperstimulation. J Assist Reprod Genet. 2002;19:507-11.

25. Nisolle M, Donnez J. Peritoneal endometriosis, ovarian endometriosis, and adenomyotic nodules of the rectovaginal septum are three different entities. Fertil Steril. 1997;68:585-96.

26. Donnez J, Lousse JC, Jadoul P, Donnez O, Squifflet J. Laparoscopic management of endometriomas using a combined technique of excisional (cystectomy) and ablative surgery. Fertil Steril. 2010;94:28-32. 
27. Baggish MS, Tucker RD. Tissue actions of bipolar scissors compared with monopolar devices. Fertil Steril. 1995;63:422-6.

28. Nezhat C, Nezhat F. Postoperative adhesion formation after ovarian cystectomy with and without ovarian reconstruction. Abstract $\mathrm{O}-012,47^{\text {th }}$ annual meeting of the American Fertility Association; Orlando, FL. 1991.

29. Operative Laparoscopy Study Group. Postoperative adhesion development after operative laparoscopy: evaluation at early second-look procedure. Fertil Steril. 1991;55:700-4.
30. Redwine DB. Conservative laparoscopic excision of endometriosis by sharp dissection: life table analysis of reoperation and persistent or recurrent disease. Fertil Steril. 1991;56:628-34.

Cite this article as: Yadav A, Patel S. A review article on ovarian cystectomy in endometriomas by laparoscopy. Int J Reprod Contracept Obstet Gynecol 2017;6:4754-8. 